International Journal of Wireless \& Mobile Networks (IJWMN) Vol. 4, No. 4, August 2012

\title{
TWO HOP CONNECTIVITY FOR UNIFORMED RANDOMLY DISTRIBUTED POINTS IN THE UNIT SQUARE
}

\author{
Johannes Karlsson \\ Embedded Systems Lab, Umeå University, Sweden \\ johannes.karlssondte.umu.se
}

\begin{abstract}
Connectivity in ad-hoc networks is a fundamental, but to a large extend still unsolved problem. In this paper we consider the connectivity problem when a number of nodes are uniformly distributed within a unit square. We limit our problem to the one-hop and two-hop connectivity. For the one-hop connectivity we calculate the exact analytically solution. For the two-hop connectivity we calculate the lower and upper bound for connectivity.
\end{abstract}

\section{KEYWORDS}

Connectivity, Ad-Hoc networks

\section{INTRODUCTION}

In wireless ad-hoc networks it is important to know the probability to have a path between two nodes for a given node density. This can easily be found using simulations but no analytically solution is available. In this paper we consider the case when a number of nodes are uniformly distributed in a unit square. The reason why we use this distribution is to make it possible to compare the results with network simulators where the nodes often are uniformly distributed within a square. We will calculate the exact analytically solution for the one hop case, when there are only two nodes, and the upper and lower bound for the two-hop case.

Some related research has been done in this area. The theory of random geometric graphs is a rather realistic model for wireless networks. In this model $n$ nodes are randomly distributed in a $\mathrm{d}$-dimensional space with a common density $\mathrm{f}$. Here the status of different edges in the graph is not independent. If $\mathrm{Xi}$ is close to $\mathrm{Xj}$, and $\mathrm{Xj}$ is close to $\mathrm{Xk}$, then $\mathrm{Xi}$ will be fairly close to $\mathrm{Xk}$. Connectivity has been studied in this type of graphs, but only for very dense graphs [1]. Feng Xue et al. are considering the number of neighbors needed for connectivity of wireless networks [2]. The nodes are uniformly and independently distributed in a unit square. They have found the number of neighbors needed for the network to be asymptotically connected. This definition does not give the answer to the probability for connectivity as a function of the node density. Leonard E. Miller has addressed a similar problem as our for two-hop connectivity [3], but in this paper he assumes Gaussian distribution between the points. In our paper we use uniform distribution. The one-hop connectivity has been solved by Miller [4]. We include our solution for one hop connectivity in this paper since we use the same approach to solve two hop connectivity.

\section{THE DISTRIBUTION FOR THE DISTANCE BETWEEN TWO RANDOM POINTS}

Let two points, $\mathrm{X}$ and $\mathrm{Y}$, be uniformly and independently distributed in the unit square, $\mathrm{K}$ (1). The ball, $B \tau(p)$, with center $p$ and radius $\tau$ is defined in (2). Let $\Gamma$ be the area of the part of the 
circle inside the unit square having the center in point $X$ and a radius of $\tau$. The probability for the distance between $\mathrm{X}$ and $\mathrm{Y}$ to be less or equal to $\tau$ is the same as the expected size of the area $\Gamma$, (3). The distance between the points can be between 0 and $\sqrt{2}$. It is however not interesting to consider connectivity for large values of $\tau$. We are therefore only solving the probability density function for $\tau$ between 0 and 1 .

$$
\begin{aligned}
& X, Y \in U(K),|K|=1 \\
& B_{T}(p)=\{y:|y-p| \leq T\}, y, p \in \Re^{2} \\
& P(d(X, Y) \leq T)=P\left(Y \in B_{T}(X)\right)=E(\Gamma)
\end{aligned}
$$

Depending on where in the unit square the point $X$ (center of the circle) is located the size of the area of the circle is calculated in different ways. If the distance from point $X$ to one edge is less than the radius then a segment of the circle will be located outside of the unit square. If the distance from point $X$ is less than the radius from two edges then two circle segments will be outside of the unit square. Otherwise the entire circle will be within the unit square. We therefore divide the unit square into three different zones, see Fig. 1.

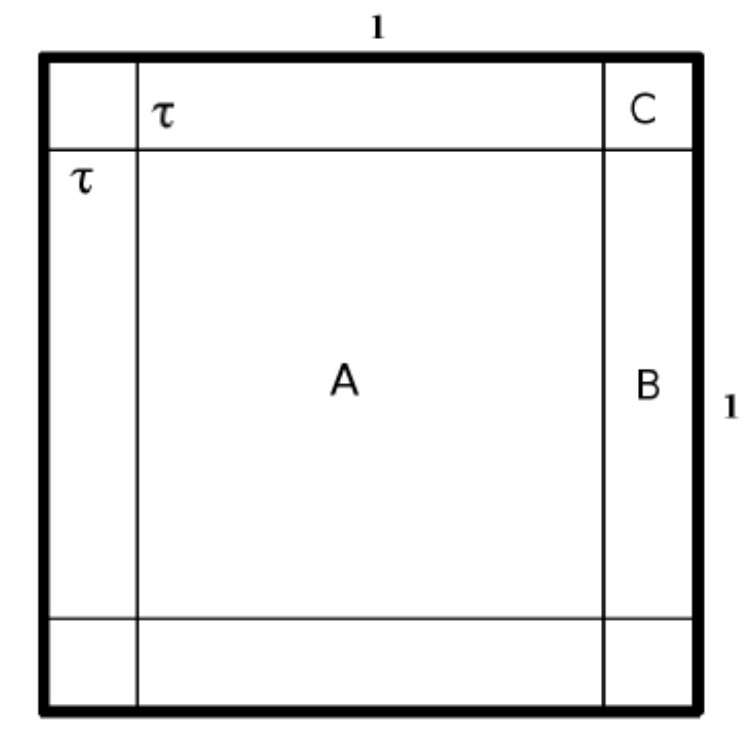

Figure 1. The different zones used in the unit square.

We then calculate the probability for point $X$ to be located in each of the zones $(4,5,6)$ and then the expected size of the area of the circle given that point $X$ is located in each zone $(7,13,19)$. The probability for point $\mathrm{X}$ to be located in each of the zones is given by:

$$
\begin{aligned}
& \mathrm{P}(X \in A)=(1-2 \mathrm{~T})^{2} \\
& \mathrm{P}(X \in \mathrm{B})=4 \mathrm{~T}(1-2 \mathrm{~T}) \\
& \mathrm{P}(X \in C)=4 \mathrm{~T}^{2}
\end{aligned}
$$

For zone A the entire circle is always within the unit square and the expected area of the circle is given by:

$$
E(\Gamma \mid X \in A)=\pi^{2}
$$


International Journal of Wireless \& Mobile Networks (IJWMN) Vol. 4, No. 4, August 2012

When point $\mathrm{X}$ is located in zone $\mathrm{B}$ a segment of the circle is outside of the unit square. We use the variable $\mathrm{n}$ for the distance between the edge and the center of the circle. This distance is uniformly distributed between 0 and $\tau$.

$$
\mathrm{n} \in[0, \mathrm{~T}]
$$

The size of the circle segment, $\mathrm{M}$, as a function of $\mathrm{n}$ is given by substituting (8) and (9) in (10):

$$
\begin{aligned}
S & =2 T \cos ^{-1}(n / T) \\
c & =2 \sqrt{T^{2}-n^{2}} \\
M & =(1 / 2)(T S-n c) \\
M= & g(n, T) \\
= & T^{2} \cos ^{-1}(n / T)-n \sqrt{T^{2}-n^{2}}
\end{aligned}
$$

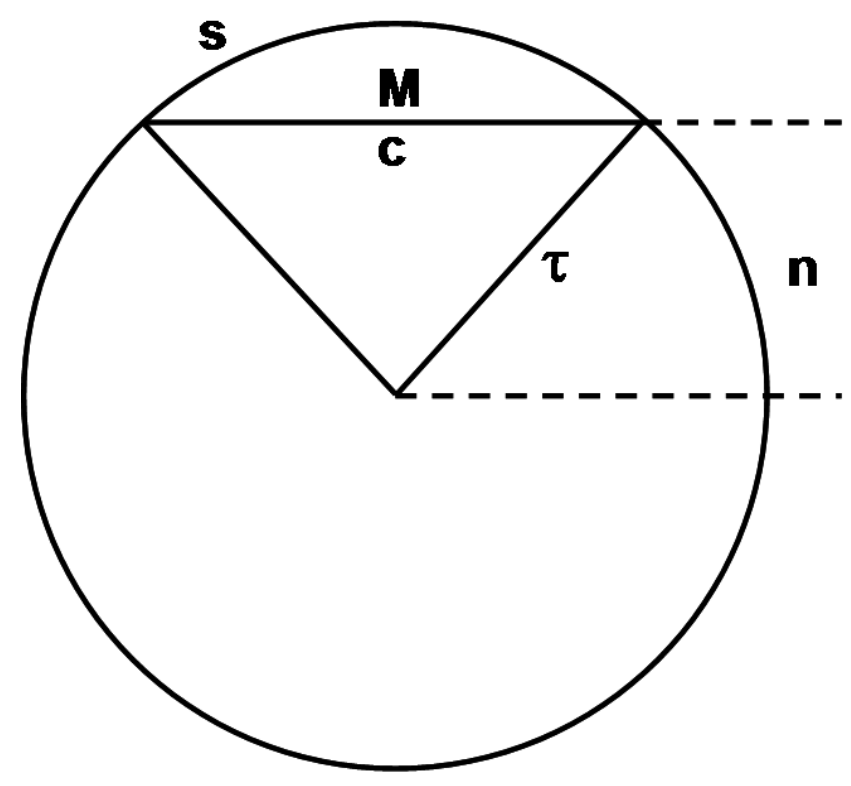

Figure 2. A segment of a circle.

The expected size of the circle segment outside of the unit square is given by:

$$
\begin{aligned}
E(M) & =E\left(g(n, T) f_{n}(n)\right) \\
& =\int_{n=0}^{T}\left(T^{2} \cos ^{-1}\left(\frac{n}{T}\right)-n \sqrt{T^{2}-n^{2}}\right) \frac{1}{T} d n \\
& =(2 / 3) T^{2}
\end{aligned}
$$

The expected size of the area inside the unit square when point $\mathrm{X}$ is located in zone $\mathrm{B}$ is given by: 
International Journal of Wireless \& Mobile Networks (IJWMN) Vol. 4, No. 4, August 2012

$$
\begin{aligned}
E(\Gamma \mid X \in B) & =\pi^{2}-E(M) \\
& =(\pi-(2 / 3)) T^{2}
\end{aligned}
$$

For zone $\mathrm{C}$ the circle can be partly located outside of two edges. For each of the edges the average size outside of the edge is given by (12).

There will be an overlapping between these areas. This is the area L in Fig. 3. The position (Q, $\mathrm{U})$ for the corner will be random and uniformly distributed (14). The area $\mathrm{L}$ will be a function of the position of the corner (15).

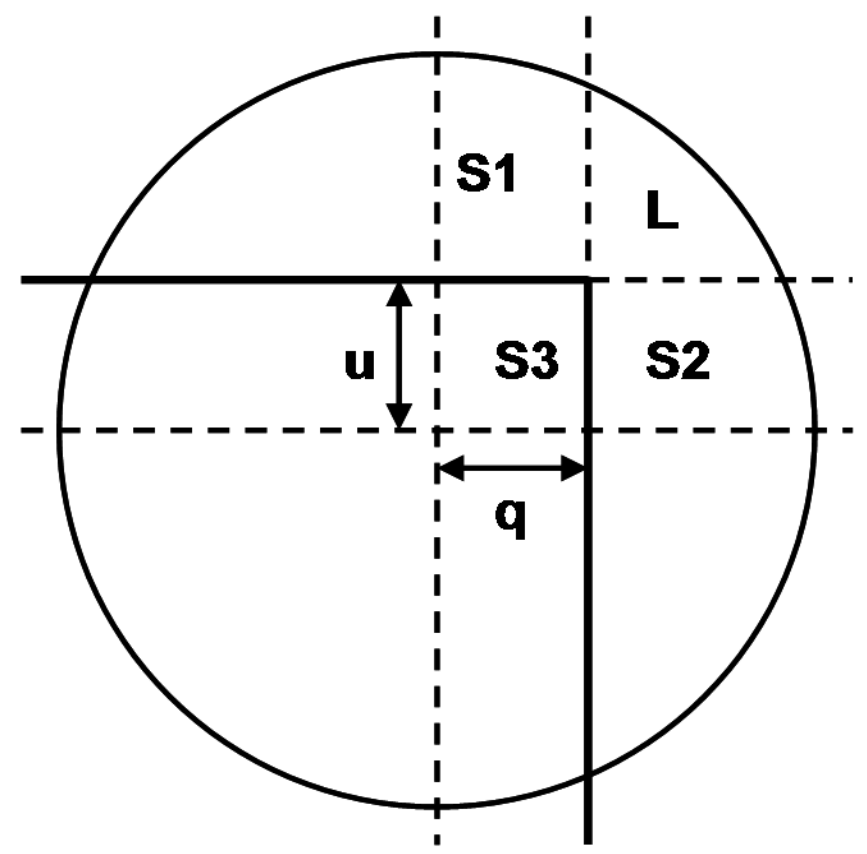

Figure 3. The overlapping area between two circle segments.

$$
\begin{aligned}
Q, U & \in[0, T] \\
L & =g(q, u) I_{1}\left(\sqrt{q^{2}+u^{2}}\right) \\
I_{1}(i) & = \begin{cases}1, & i \leq T \\
0, & i>T\end{cases}
\end{aligned}
$$

We first calculate the function $\mathrm{g}(\mathrm{q}, \mathrm{u})$ in (16). This is the area $(\mathrm{S} 1+\mathrm{L})+(\mathrm{S} 2+$ $\mathrm{L})+(\mathrm{S} 3)-(\mathrm{S} 1+\mathrm{S} 2+\mathrm{S} 3+\mathrm{L})$. The areas $(\mathrm{S} 1+\mathrm{L})$ and $(\mathrm{S} 2+\mathrm{L})$ are half circle segments and these areas are found by using (11). The expected size of $\mathrm{L}$ is then found in (18). The pdf for (q, u) is given in (17). 
International Journal of Wireless \& Mobile Networks (IJWMN) Vol. 4, No. 4, August 2012

$$
\begin{aligned}
& g(q, u)= \frac{T^{2} \cos ^{-1}(q / T)-q \sqrt{T^{2}-q^{2}}}{2} \\
&+\frac{T^{2} \cos ^{-1}(u / T)-u^{\sqrt{T^{2}-u^{2}}}}{2} \\
&+q u-\frac{m^{2}}{4} \\
& f_{q u}(q, u)= 1 / T^{2} \\
& E(L)=\int_{q=0}^{T} \int_{u=0}^{v} \overline{T^{2}-q^{2}} g(q, u) f_{q u}(q, u) d u d q \\
&=(1 / 8) T^{2}
\end{aligned}
$$

The expected size of the area inside the unit square when point $\mathrm{X}$ is located in zone $\mathrm{C}$ is given by:

$$
\begin{aligned}
E(\Gamma \mid X \in C) & =\pi^{2}-(E(M)-E(L)) \\
& =\pi^{2}-\left(2(2 / 3) T^{2}-(1 / 8) T^{2}\right) \\
& =\pi^{2}-(29 / 24) T^{2}
\end{aligned}
$$

The total contributed expected size from each of the three areas is given by multi- plying the probability for being in that area with the expected size when the center of the circle is located inside of each of the areas.

$$
\begin{aligned}
& E(\Gamma \mid X \in A) P(X \in A) \\
& =(1-2 T)^{2} \Pi^{2} \\
& =\pi^{2}-4 \pi^{3}+4 \pi \pi^{4} \\
& E(\Gamma \mid X \in B) P(X \in B) \\
& =4 T(1-2 T)\left(\pi^{2}-(2 / 3) T^{2}\right) \\
& =4 \pi^{3}-8 \pi T^{4}-(8 / 3) T^{3}+(16 / 3) T^{4} \\
& E(\Gamma \mid X \in C) P(X \in C) \\
& =4 T^{2}\left(\pi^{2}-(29 / 24) T^{2}\right) \\
& =4 \pi^{4}-(29 / 6) T^{4}
\end{aligned}
$$

By adding (21), (22) and (23) we will have the expected area of a circle having radius $\tau$ with the center uniformly distributed inside a unit square. This is also the probability for two randomly uniformly distributed points to have a distance less or equal to $\tau$. This is the cumulative probability distribution function (cdf) for the distance between to random points in the unit square. (24).

$$
F(T)=(1 / 2) T^{4}-(8 / 3) T^{3}+\pi T^{2}
$$

The pdf is given in (25) by taking the derivative of the cdf. 
International Journal of Wireless \& Mobile Networks (IJWMN) Vol. 4, No. 4, August 2012

$$
f(T)=2 T^{3}-8 T^{2}+2 \pi T
$$

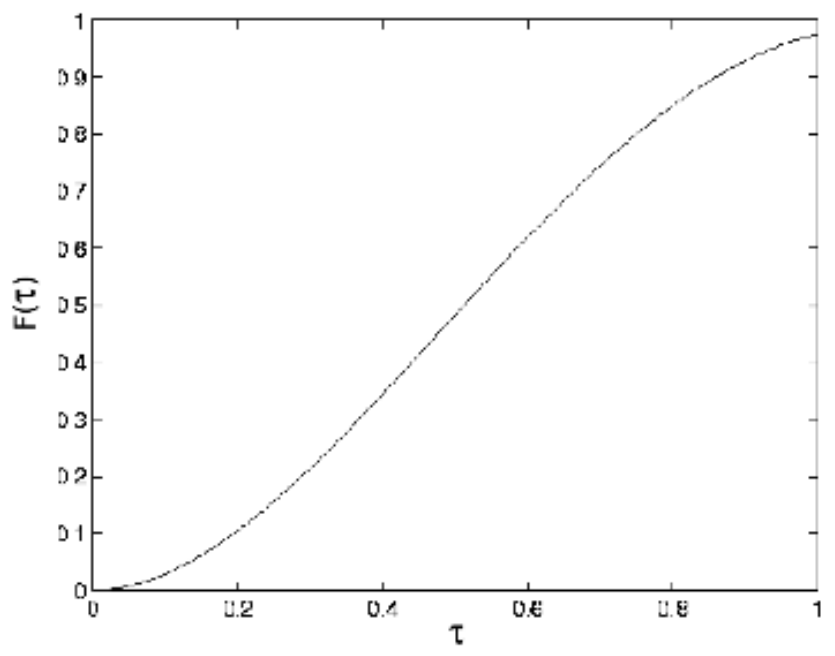

Figure 4. The cdf for the distance between two random points in the unit square.

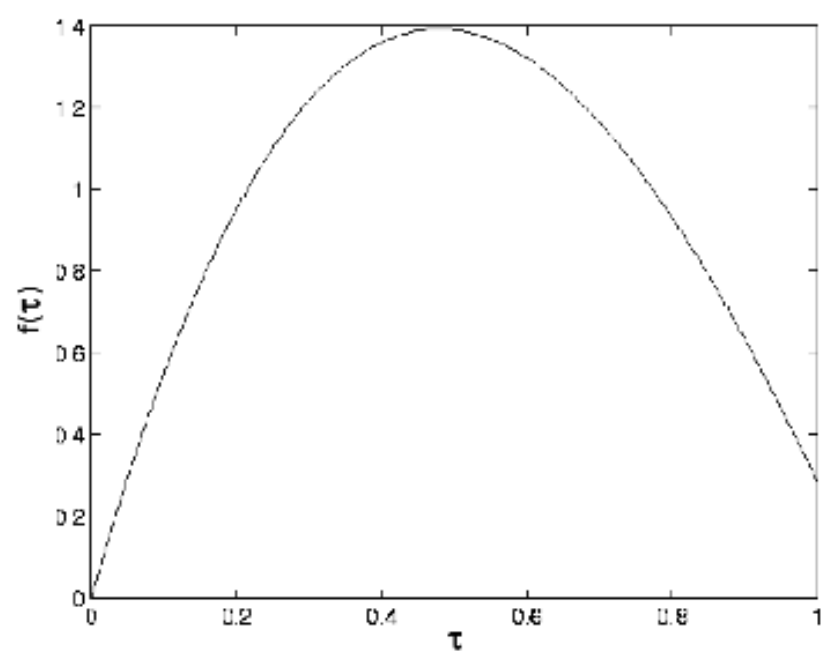

Figure 5. The pdf for the distance between to random points in the unit square.

\section{PROBABILITY FOR TWO HOP CONNECTION}

Consider three nodes uniformly and randomly distributed in the unit square, see Fig. 6.

$$
X, Y, Z \in U(K),|K|=1
$$


International Journal of Wireless \& Mobile Networks (IJWMN) Vol. 4, No. 4, August 2012

What is the probability that the distance $\mathrm{X}$ to $\mathrm{Y}$ and $\mathrm{Y}$ to $\mathrm{Z}$ is less than a threshold $\tau$ and the distance $\mathrm{X}$ to $\mathrm{Z}$ is larger than the same threshold $\tau$ ? In other words; what is the probability for having a two hop connection between $\mathrm{X}$ and $\mathrm{Z}$ and at the same time not an one hop connection for a given max hop distance $\tau$ ? (26) We use I.I to describe the area covered by a set.

$$
\begin{aligned}
P & =P(d(X, Y) \leq \tau \cap d(Y, Z) \leq \tau \cap d(X, Z) \geq \tau) \\
& =E_{X, Z} P\left(Y \in\left(B_{\tau}(X) \cap B_{\tau}(Z)\right)\right) I_{2}(d(X, Z)) \\
& =E_{X, Z}\left|B_{\tau}(X) \cap B_{\tau}(Z) \cap K\right| I_{2}(d(X, Z)) \\
I_{2}(v) & = \begin{cases}1, & v \geq \tau \\
0, & v<\tau\end{cases}
\end{aligned}
$$

The probability for having a two hop connection and at the same time not an one hop connection is the overlapping between two circles when the distance between the center of these circles is between $\tau$ and $2 \tau$, we use P for this probability (26). It is however rather difficult to calculate the exact function for this value. In this paper we will therefore calculate the upper and lower bound for $\mathrm{P}$.

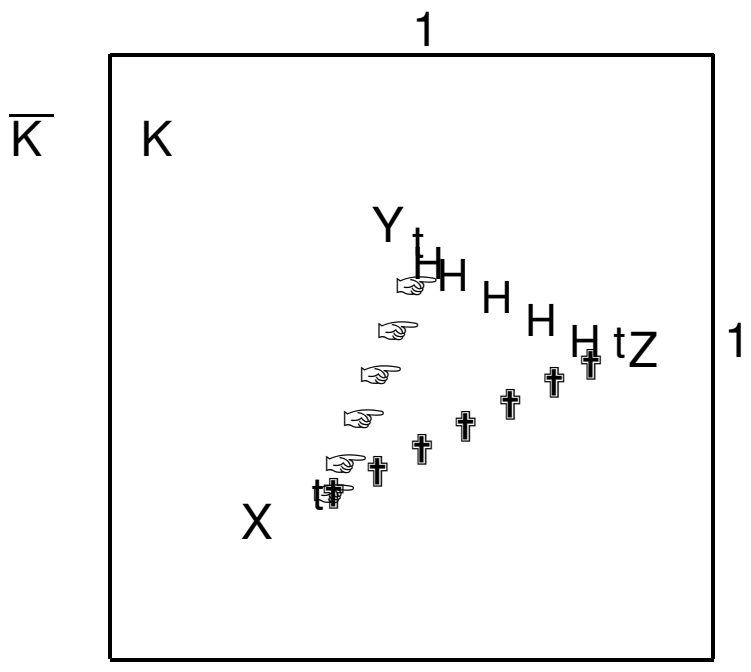

Figure 6. Three points uniformly distributed in the unit square.

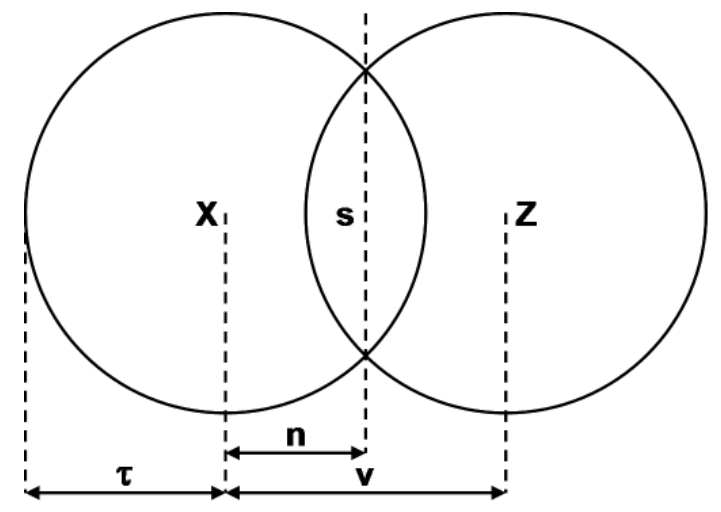

Figure 7. The overlapping area between the coverage of two nodes. 


\subsection{Upper bound}

For the upper bound we use the entire overlapping between the circles, both the part that is inside of the unit square and the part that is outside of the unit square (28). The size of the intersection as a function of the distance between the center of the two circles is given by (27). The expected size of the area is given in (29) and is found by integrating (27) multiplied with the pdf (25) for the distance over the valid distances.

$$
\begin{aligned}
& B_{1}(X, Z)=B_{T}(X) \cap B_{T}(Z) \\
f_{s}(v)= & 2\left(T^{2} \cos ^{-1}\left(\frac{v}{2 T}\right)-\frac{v}{2} \sqrt{T^{2}-\left(\frac{v}{2}\right)^{2}}\right) \\
P= & E_{X, Z}\left|B_{1}(X, Z) \cap K\right| I_{2}(d(X, Z)) \\
\leq & E_{X, Z}\left|B_{1}(X, Z)\right| I_{2}(d(X, Z))=P_{u} \\
P_{u}= & E_{X, Z} f_{s}(d(X, Z)) I_{2}(d(X, Z)) \\
= & \int_{v=T}^{2 T} f_{s}(v) f(v) d v \\
= & \int_{v=T}^{2 T} 2\left(T^{2} \cos ^{-1}\left(\frac{v}{2 T}\right)-\frac{v}{2} \sqrt{T^{2}-\left(\frac{v}{2}\right)^{2}}\right) \\
& \cdot\left(2 v^{3}-8 v^{2}+2 \pi v\right) d v \\
= & \left((\pi / 3)+\left(\left(3^{3} \overline{3}\right) / 4\right)\right) T^{6} \\
& +\left(\frac{16 \pi}{9}-\frac{36 \sqrt{3}}{5}\right) T^{5}+\left(\frac{3 \pi}{4}\right) T^{4}
\end{aligned}
$$

\subsection{Lower bound}

The probability for $\mathrm{P}$ is given by the total overlapping between the circles (30) minus the part of the overlapping that intersects with the area outside of the unit square (31). To calculate the lower bound for the probability $\mathrm{P}$ we will calculate a function, $\mathrm{g}_{\mathrm{u}}(\tau)$, that is greater or equal to the part of the overlapping intersecting with the area outside of the unit square (32).

$$
\begin{aligned}
f(T) & =E_{X, Z}\left|B_{1}(X, Z)\right| I_{2}(d(X, Z)) \\
g(T) & =E_{X, Z}\left|B_{1}(X, Z) \cap \bar{K}\right| I_{2}(d(X, Z)) \\
P & =f(T)-g(T) \\
g_{u}(T) & \geq g(T) \\
P_{l} & =f(T)-g_{u}(T) \leq P
\end{aligned}
$$


International Journal of Wireless \& Mobile Networks (IJWMN) Vol. 4, No. 4, August 2012

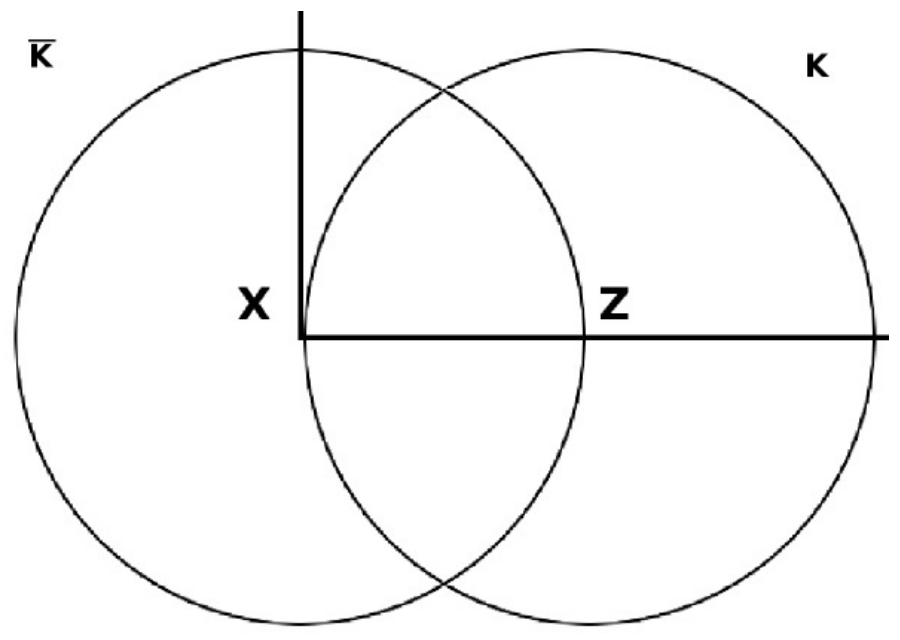

Figure 8 . The point $\mathrm{X}$ is located in a corner and the point $\mathrm{Z}$ is located on an edge.

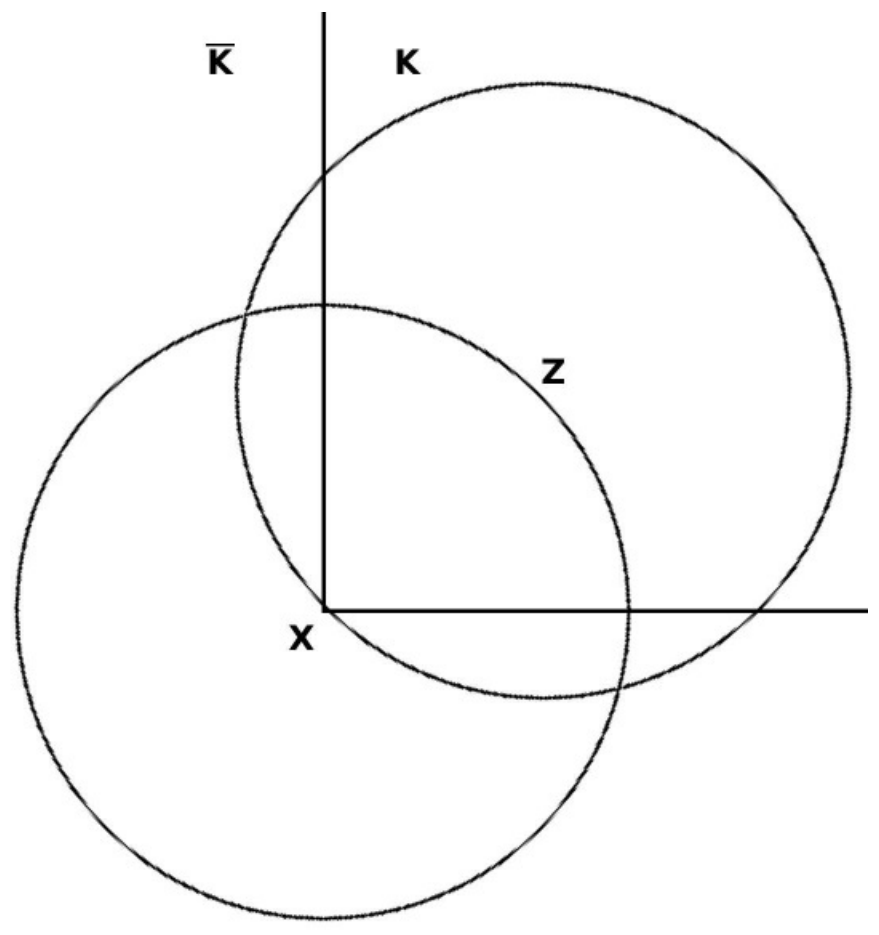

Figure 9. Less than half of the overlapping outside of the unit square.

It is only possible for $\mathrm{B}_{1}(\mathrm{X}, \mathrm{Z})$ to intersect with $\mathrm{K}$ if $\mathrm{B}_{\tau}(\mathrm{X})$ and $\mathrm{B}_{\tau}(\mathrm{Z})$ also intersects with $\mathrm{K}$. The probability for this is given in (33). For these cases we put the point $\mathrm{X}$ at the location giving the largest average value for $\mathrm{B}_{1}(\mathrm{X}, \mathrm{Z}) \cap \mathrm{K}$; this is when $\mathrm{X}$ is located in one corner of the unit square. The probability density function for the distance to point $\mathrm{Z}$ given that point $\mathrm{X}$ is located in the corner is given in (34). 
International Journal of Wireless \& Mobile Networks (IJWMN) Vol. 4, No. 4, August 2012

$$
\begin{aligned}
\mathrm{P}_{\mathrm{o}}(\mathrm{T}) & =\mathrm{P}\left(\left(\mathrm{B}_{\mathrm{T}}(\mathrm{X}) \nsubseteq \mathrm{K}\right) \cap\left(\mathrm{B}_{\mathrm{T}}(\mathrm{Z}) \nsubseteq \mathrm{K}\right)\right) \\
& =(4 \mathrm{~T}(1-\mathrm{T}))^{2} \\
\mathrm{f}_{\mathrm{V}}(\mathrm{V}) & =\frac{\pi \mathrm{V}}{2}
\end{aligned}
$$

For all distances between $\mathrm{X}$ and $\mathrm{Z}$ we set the area outside of the unit square to the highest amount possible. That is half of the total overlapping (35). These two assumptions are based on extensive simulations. The expected size of this area is solved in (36) by using (27), (34), and (33).

$$
\begin{aligned}
g_{u}(T)= & P_{0}(T) \frac{1}{2} E_{Z}\left|B_{1}(X, Z)\right| I_{2}(d(X, Z)) \\
g_{u}(T)= & (4 T(1-T))^{2} \\
& \cdot \int_{V=T}^{2 T}\left(T^{2} \cos ^{-1}\left(\frac{v}{2 T}\right)-\frac{v}{2} \sqrt{T^{2}-\left(\frac{V}{2}\right)^{2}}\right) \\
& \cdot\left(\frac{\pi V}{\sqrt{2}}\right) d V \\
= & \frac{3 \overline{3} \pi(1-T)^{2} T^{6}}{2}
\end{aligned}
$$

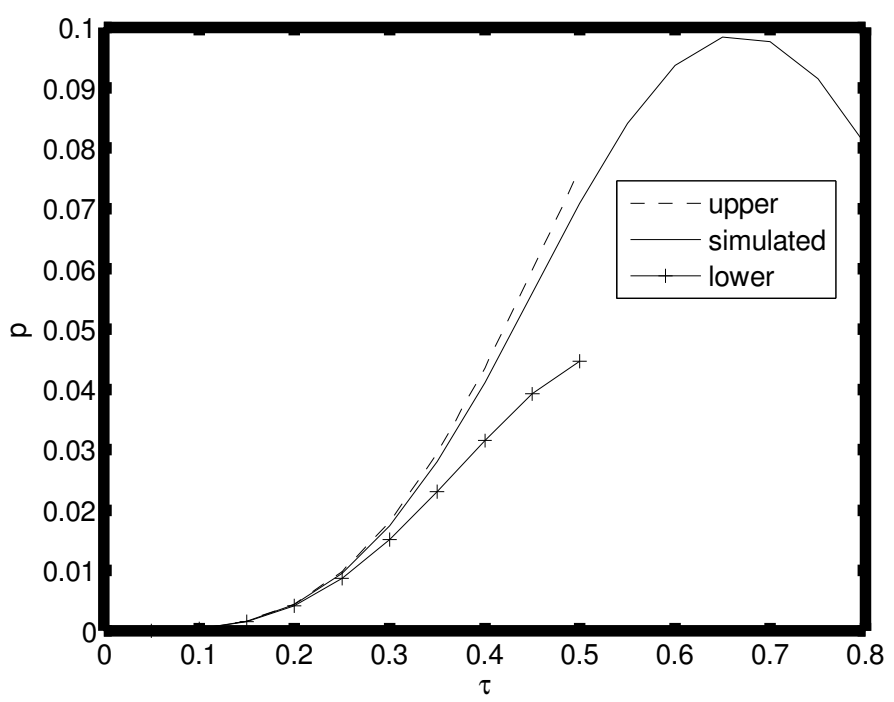

Figure 10. The lower and upper bound compared to the simulated result. 


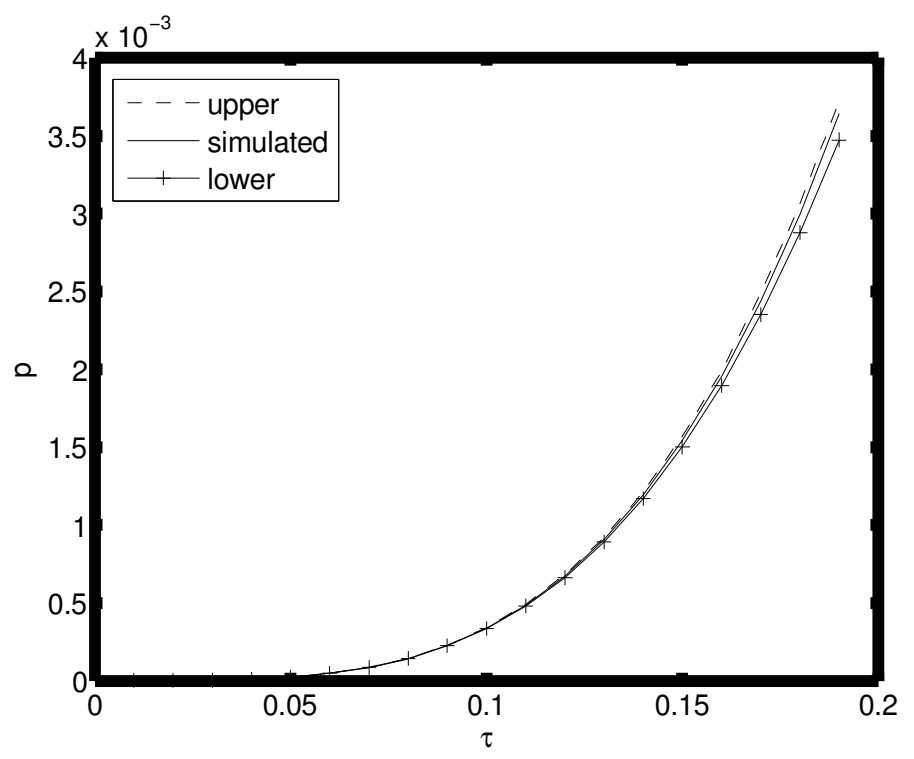

Figure 11. The lower and upper bound compared to the simulated result.

The upper bound for the probability of having a two hop connection between two random points in the unit square is given in (37). The lower bound is given in (38).

$$
\begin{aligned}
& P_{u}(T)=\left(\frac{\pi}{3}+\frac{3^{V} \overline{3}}{4}\right) T^{6}+\left(\frac{16 \pi}{9}-\frac{36^{\sqrt{ }} \overline{3}}{5}\right) T^{5}+\left(\frac{3 \pi^{V} \overline{3}}{4}\right) T^{4} \\
& P_{I}(T)=\left(\frac{\pi}{3}+\frac{3^{\sqrt{3}}}{4}\right) T^{6}+\left(\frac{16 \pi}{9}-\frac{36^{\sqrt{3}}}{5}\right) T^{5}+\left(\frac{3 \pi}{4}\right) T^{4} \\
& -\frac{3^{\sqrt{3} \pi(1-T)^{2} T^{6}}}{2}
\end{aligned}
$$

\section{Conclusions}

In this paper we have presented an exact function for the one-hop connectivity and a lower and upper bound for the two-hop connectivity. These results have been compared to simulated results for the two-hop connectivity. In future work the upper and lower bounds can be improved. It can also be possible to find the exact solution by making use of the joint distribution for the distance between the three points. 
International Journal of Wireless \& Mobile Networks (IJWMN) Vol. 4, No. 4, August 2012

\section{REFERENCES}

[1] Paolo Santi and Douglas M. Blough, "The critical transmitting range for connectivity in sparse wireless ad hoc networks," IEEE Transactions on Mobile Computing, vol. 2, no. 1, pp. 25-39, 2003.

[2] Feng Xue and P. R. Kumar, "The number of neighbors needed for connectivity of wireless networks," Wirel. Netw., vol. 10, no. 2, pp. 169-181, 2004.

[3] Leonard E. Miller, "Probability of a two-hop connection in a random mobile network," in Proceedings 35th Conference on Information Sciences and Systems, Baltimore, March 2001.

[4] Leonard E. Miller, "Distribution of link distances in a wireless network, " Journal of Research of the National Institute of Standards and Technology, vol. Volume 106, no. Number 2, pp. 401412, March April 2001.

\section{Authors}

Johannes Karlsson is an Associate Professor and the Director of Embedded Systems Laboratory, Department of Applied Physics and Electronics, Umea University, Sweden. He has been the project leader for several large scale EU projects such as "Digital Zoo" and "Animal Sensor Networks". His current research focus is around multimedia signal processing and transportation in wireless sensor networks and energy management in large scale environmental sensor networks. He received his PhD degree in applied electronics from Umea University, Sweden, in 2010. The thesis dealt with real-time video communication over wireless ad-hoc networks. For this work he was selected as

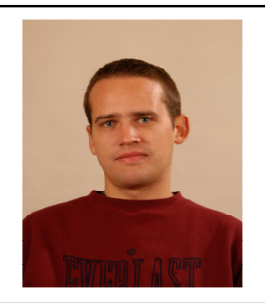
a finalist for the prestigious Chester Carlson prize. 